This is the final peer-reviewed accepted manuscript of:

S. Lilla, C. Orozco, A. Borghetti, F. Napolitano and F. Tossani, "Day-Ahead Scheduling of a Local Energy Community: An Alternating Direction Method of Multipliers Approach," in IEEE Transactions on Power Systems, vol. 35, no. 2, pp. 1132-1142, March 2020.

The final published version is available online at:

https://doi.org/10.1109/TPWRS.2019.2944541

Rights / License:

The terms and conditions for the reuse of this version of the manuscript are specified in the publishing policy. For all terms of use and more information see the publisher's website. 


\title{
Day-ahead Scheduling of a Local Energy Community: An Alternating Direction Method of Multipliers Approach
}

\author{
Stefano Lilla, Student Member, IEEE, Camilo Orozco, Student Member, IEEE, A. Borghetti, Fellow, \\ IEEE, F. Napolitano, Senior Member, IEEE, F. Tossani, Member, IEEE
}

\begin{abstract}
The paper focuses on the day-ahead operational planning of a grid-connected local energy community (LEC) consisting of an internal low-voltage network and several prosumers including generation units, battery storage systems, and local loads. In order to preserve, as much as possible, the confidentiality of the features of prosumers' equipment and the production and load forecasts, the problem is addressed by designing a specific distributed procedure based on the alternating direction method of multipliers (ADMM). The distributed procedure calculates the scheduling of the available energy resources to limit the balancing action of the external grid and allocates the internal network losses to the various power transactions. Results obtained for various case studies are compared with those obtained by a centralized optimization approach. The results confirm that, in the considered LEC framework, each of the prosumers achieves a reduction in costs or increases revenues in case it participates to the LEC with respect to the case in which it can only transact with an external energy provider.
\end{abstract}

Index Terms - alternating direction method of multipliers, distributed optimization, energy management system, local energy community, mixed integer linear programming, mixed integer quadratic programming.

\section{INTRODUCTION}

$\mathrm{W}$ E consider a local energy community (LEC), i.e., a set of residential or small industrial sites, each acting as a prosumer and generally including generation and battery energy storage (BES) units as well as loads. The LEC has an internal low voltage (LV) distribution network, which is connected to the external utility grid. In a LEC, each prosumer uses the available energy resources in cooperation with the other prosumers to minimize the energy procurement cost of the entire LEC.

The LEC definition adopted in this paper is characterized by

This work was supported in part by the Italian Ministry of Economic Development in the framework of CERSE research project PODCAST and by the European Union's Horizon 2020 research and innovation programme under the Marie Sklodowska-Curie grant agreement No 675318 (INCITE) and the Electronic Components and Systems for European Leadership Joint Undertaking under grant agreement No 737434 (CONNECT).

The authors are with the Department of Electrical, Electronic and Information Engineering, University of Bologna, 40136 Bologna, Italy (email: \{stefano.lilla3,camilo.orozco2,alberto.borghetti,fabio.napolitano,fabio. tossani\}@unibo.it). being local and cooperative: all the prosumers are connected to the same low voltage distribution network and they collaborate without any competitive strategy (i.e., without exploiting their market power) for the common goal of minimizing the costs due to the exchanges with the utility grid. These characteristics makes the studied framework quite different from those dealt with in, e.g., [1], [2], and references therein, which consider competitive markets where the various operators act with different objectives in a noncooperative scheme. The community concept can be more general, particularly if there is the possibility for a prosumer to join another local trading platform, a possibility that is not considered in this paper.

In the literature, there are several studies regarding real implementations of the LEC concept, e.g. the Brooklyn microgrid project [3]. The economic justification for the formation of a LEC is mainly due to the difference between the price of the energy supplied by the external energy provider and the price sold by the LEC to the utility grid. This difference can be significant, e.g., due to the costs of the ancillary services.

The operation of a LEC requires the implementation of an energy management system (EMS) for the optimal exploitation of the available resources [4]. This paper focuses on the EMS function that provides the day-ahead scheduling of the BES units, under the assumption that all the generation units of the LEC are photovoltaic (PV) systems. The scheduling function is structured as a distributed optimization algorithm based on the alternating direction method of multipliers (ADMM). The main inputs of the plans of each prosumer are the forecasts of the photovoltaic production and the load. The prosumers' decisions are coordinated by the procedure that iteratively updates the multipliers. This paper does not address the issues of the uncertainty associated with the forecasts, as accomplished, for instance, in [5].

The scheduling function can be also structured as a centralized optimization problem, in which a control unit collects and keeps updated all the characteristics of the prosumers' equipment and all the load and PV production forecasts. Compared to a centralized approach, the use of a distributed approach, like ADMM, limits the information that every prosumer needs to communicate. Indeed, the procedure for the update of multipliers only requires the knowledge of the energy exchange between prosumers. Furthermore, a distributed procedure is more appropriate for the 
implementation of new transaction methods based, e.g., on blockchain [6], [7], or, more generally, on distributed ledger technologies.

This paper describes first the centralized optimization approach based on a mixed integer linear programming (MILP) model and then it focuses on the ADMM-based one.

ADMM is one of the most frequently adopted consensus algorithms (see, e.g., [8]-[11], and references therein) and it has been recently investigated for the solution of scheduling problems in microgrids and for the more general problem of the optimal operation of multi-microgrids and active distribution networks. Both [12] and [13] deal systems similar to the one considered in this paper, with the presence of local generation and BES units and with the possibility of exchanging energy with an external utility grid, but they neglect line-losses in the optimization procedure. In [13] the uncertainty of renewable energy production, load consumption, and energy prices is addressed through a robust optimization approach, whilst [14] applies a regret minimization.

In [15] the use of a primal Benders decomposition approach instead of Lagrangian-based dual decomposition, such as the ADMM, has been presented. Other approaches adopt hierarchical architectures with a central controller or community manager that coordinates the power transaction among prosumers or microgrids and the trading with the utility grid, as described in, e.g., [16], [17] and references therein.

The specific characteristics of the distributed procedure proposed in this paper are:

- it aims at minimizing the energy procurement cost of the LEC considering the power loss in the internal network;

- the internal network losses are allocated to each energy transaction between two prosumers or between a prosumer and the utility grid;

- the results obtained by the distributed algorithm are compared with those given by a centralized model that includes the same constraints and power loss allocation;

- the structure of the proposed scheduling procedure is consistent with the billing procedure and the metering systems typically installed in a LEC.

The structure of the paper is the following. Section II is devoted to the formulation of the problem and the description of a centralized approach based on a MILP model. Section III presents the formulation of the proposed distributed procedure based on an ADMM algorithm. Section IV compares the results of numerical tests provided by both the above-mentioned approaches. Section V concludes the paper.

\section{PROBlem Formulation - CENTRALIZED APPROACH}

Fig. 1 illustrates the scheme of the LEC. The grid meter $\mathbf{M}_{\mathrm{g}}$, positioned at the point of common coupling with the external utility grid, is bidirectional and measures the energy exchanged in each time interval.

For the implementation of the distributed optimization approach, each prosumer $i$ is equipped with a local bidirectional meter $\mathrm{M}_{\mathrm{i}}$ that measures the energy that the specific prosumer exchanges with the internal network in each time interval. Given the collaborative characteristic of the LEC, a prosumer cannot act as producer and as consumer in the same time interval.

The day ahead scheduling dealt with in this paper provides a

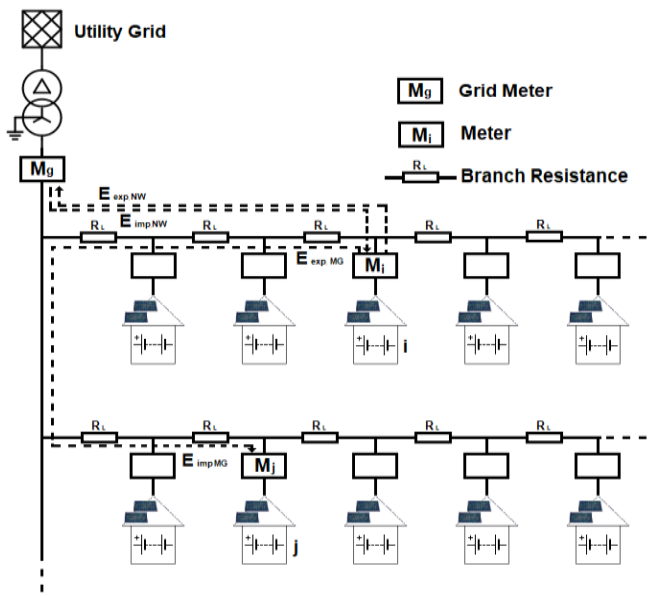

Fig. 1. Scheme of a LEC with the internal network, prosumers, and meters (adapted from [25]).

plan of the optimal use of the LEC energy resources during the following day, with particular reference to the BES units, and calculates the prices of the energy transactions between prosumers. It is assumed that the prices of exchanges with the utility grid are predefined, although they vary according to the time of day.

The electricity billing procedure can be described as follows:

a) In each time interval, if the LEC buys energy from the utility grid (measured by $\mathrm{M}_{\mathrm{g}}$ ), the relevant cost is allocated to each consumer $i$ (i.e., to each prosumer who consumes energy in excess of the local generation in that time interval) proportionally to the ratio between its consumption measured by $\mathrm{M}_{\mathrm{i}}$ and the total consumption in the LEC, i.e., the sum of the measured energies by all the prosumers acting as consumers.

b) If the LEC sells energy to the utility grid (measured by $\mathrm{M}_{\mathrm{g}}$ ), the corresponding revenue is allocated to each producer $j$ (i.e., to each prosumer that produces energy in excess of the local load in that time interval) proportionally to the contribution of $j$ to the total LEC production, i.e., to the ratio between the energy measured by $\mathrm{M}_{\mathrm{j}}$ and the sum of the measurements of all the prosumers acting as producers.

c) Each consumer $i$ is also charged for the energy bought from the producers of the LEC, i.e., for the difference between the measurement of $\mathrm{M}_{\mathrm{i}}$ and the energy allocated to consumer $i$ in step a). The corresponding revenue of producer $j$ is estimated proportionally to the contribution of $j$ to the total LEC production as in step b). The day ahead scheduling procedure calculates the energy prices of each producer $j$ in each time interval.

Both the centralized and distributed approach are divided in two stages. Initially, the optimization is performed by using a first estimation of the power loss separately for each prosumer. Then, in the second stage, the network power loss due to all transactions is recalculated and allocated to each power transaction to perform a second optimization. 


\section{A. First stage}

By denoting as $\Omega=\{1,2, \ldots, N\}$ the set of prosumers $i$, $T=\left\{1,2, \ldots, t_{\text {end }}\right\}$ the set of intervals $t$ of the time horizon, and $B=\left\{1,2, \ldots, b_{\text {end }}\right\}$ the set of branches of the network, the centralized LEC scheduling is described by the following MILP model.

Objective function $O F(1)$ minimizes the total cost due to the power transactions with the utility grid during the following day: parameters $\pi_{b u y}^{t}$ and $\pi_{\text {sell }}^{t}$ are the prices (in $€ / \mathrm{kWh}$ ) of the energy that the LEC buys from and sells to the utility grid, respectively; $P_{\text {buy_Grid } i}^{t}$ and $P_{\text {sel__Grid } i}^{t}$ are the power (in $\mathrm{kW}$ ) bought from and sold to the utility grid, respectively; parameter $\Delta t$ is the time step (in h).

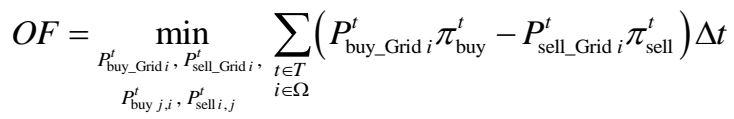

The constraints are:

$$
\begin{aligned}
& P_{\text {sell } i, j}^{t}-P_{\text {buy } j, i}^{t}=0 \quad t \in T, i, j \in \Omega \\
& P_{\mathrm{G} i}^{t}+P_{\mathrm{BES} \_ \text {dis } i}^{t}+P_{\text {buy_Grid } i}^{t}+\sum_{\substack{j \in \Omega \\
j \neq i}} P_{\text {buy } i, j}^{t}=P_{\mathrm{D} i}^{t}+P_{\mathrm{BES} \_ \text {ch } i}^{t} \\
& +P_{\text {sell_Grid } i}^{t}+\sum_{\substack{j \in \Omega \\
j \neq i}} P_{\text {sell } i, j}^{t}+\frac{1}{2} \sum_{b \in B} L_{b, i}^{t} \\
& \left\{\begin{array}{lr}
P_{\text {buy_Grid } i}^{t}=0 \text { and } P_{\text {buy } i, j}^{t}=0 \text { if } u_{i}^{t}=0 & u_{i}^{t} \in\{1,0\} \\
P_{\text {sell_Grid } i}^{t}=0 \text { and } P_{\text {sell } i, j}^{t}=0 \text { if } u_{i}^{t}=1 & i \text { and } j \in \Omega
\end{array}\right. \\
& 0 \leq P_{\text {buy_Grid } i}^{t} \leq P_{\text {buy } i}^{\max } \quad 0 \leq P_{\text {sel__Grid } i}^{t} \leq P_{\text {sell } i}^{\max } \quad t \in T, i \in \Omega \\
& 0 \leq P_{\text {buy } i, j}^{t} \leq P_{\text {buy } i}^{\max } \quad 0 \leq P_{\text {sell } i, j}^{t} \leq P_{\text {sell } i}^{\max } \quad t \in T, i \text { and } j \in \Omega \\
& E_{\mathrm{BES} i}^{t}=E_{\mathrm{BES} i}^{t-1}+\left(P_{\mathrm{BES} \_\mathrm{ch} i}^{t} \eta_{\mathrm{ch} i}-P_{\mathrm{BES} \_ \text {dis } i}^{t} / \eta_{\text {dis } i}\right) \Delta t \quad i \in \Omega \\
& \left\{\begin{array}{l}
E_{\mathrm{BES} i}^{t=1}=E_{\mathrm{BES} i}^{\max }+\left(P_{\mathrm{BES} \_ \text {ch } i}^{t=1} \eta_{\mathrm{ch} i}-P_{\mathrm{BES} \_ \text {dis } i}^{t=1} / \eta_{\mathrm{dis} i}\right) \Delta t \quad i \in \Omega \\
E_{\mathrm{BES} i}^{t_{\text {end }}}=E_{\mathrm{BES} i}^{\max } \quad i \in \Omega
\end{array}\right. \\
& \left\{\begin{array}{llll}
P_{\mathrm{BES} \_\mathrm{c} i}^{t}=0 & \text { if } & u_{\mathrm{BES} i}^{t}=0 & u_{\mathrm{BES} i}^{t} \in\{1,0\} \\
P_{\mathrm{BES} \_ \text {dis } i}^{t}=0 & \text { if } & u_{\mathrm{BES} i}^{t}=1 & i \in \Omega
\end{array}\right. \\
& 0 \leq P_{\mathrm{BES} \_ \text {dis } i}^{t} \leq P_{\mathrm{BES} i}^{\max } \quad 0 \leq P_{\mathrm{BES} \_ \text {ch } i}^{t} \leq P_{\mathrm{BES} i}^{\max } t \in T, i \in \Omega \\
& E_{\mathrm{BES} i}^{\min } \leq E_{\mathrm{BES} i}^{t} \leq E_{\mathrm{BES} i}^{\mathrm{max}} t \in T, i \in \Omega .
\end{aligned}
$$

Constraint (2) represents the equilibrium between nonnegative variable $P_{\text {buy } j, i}^{t}$, which is the power (in $\mathrm{kW}$ ) bought by $j$ from $i$ in time period $t$, and non-negative variable $P_{\text {sell } i, j}^{t}$, which is the power (in $\mathrm{kW}$ ) sold by $i$ to $j$. Constraint (2) couples all the sales transactions between prosumer $i$ and the other prosumers so that the price is the same for all the sale transaction of prosumer $i$ in time interval $t$.

Constraint (3) represents the power balance for the $i$-th prosumer in time interval $t$ : parameters $P_{\mathrm{G} i}^{t}$ and $P_{\mathrm{D} i}^{t}$ are the forecasts of PV generation and load demand (in $\mathrm{kW}$ ), respectively; non-negative variables $P_{\mathrm{BES} \_ \text {ch } i}^{t}$ and $P_{\mathrm{BES} \text { _dis } i}^{t}$ are the charge and discharge power of the BES (in $\mathrm{kW}$ ), respectively. $L_{b, i}^{t}$ are the losses in branch $b$ due to transactions involving prosumer $i$, as described later in this subsection.

Indicator constraints (4), with binary variable $u_{\mathrm{i}}^{t}$, are used to avoid simultaneous purchases and sales by the same prosumer.

In each time interval $t$, purchases and sales are limited by constraints (5) and (6), where $P_{\text {sell } i}^{\max }$ is the largest value between 0 and $P_{\mathrm{G} i}^{t}-P_{\mathrm{D} i}^{t}+P_{\mathrm{BES} i}^{\max } ; P_{\mathrm{buy} i}^{\max }$ is the largest value between 0 and $P_{\mathrm{D} i}^{t}-P_{\mathrm{G} i}^{t}+P_{\mathrm{BES} i}^{\max } ; P_{\mathrm{BES} i}^{\max }$ is the maximum power output of the BES of prosumer $i$.

The state of the energy ( $\mathrm{SoE}$ ) of the $i$-th BES unit is defined by (7) and (8), where $E_{\mathrm{BES} i}^{t}$ is the SoE at time $t$ (in $\mathrm{kWh}$ ); $E_{\mathrm{BES} i}^{\max }$ is the capacity; $\eta_{\mathrm{ch} i}$ and $\eta_{\mathrm{dis} i}$ are positive numbers lower than 1 and represent the efficiencies during charge and discharge, respectively. In (8) we assume that BES units are fully charged at the beginning and at the end of the day. Constraint (10) limits the discharge and charge power within the maximum value $P_{\mathrm{BES} i}^{\max }$. The SoE is bounded between minimum level $E_{\mathrm{BES} i}^{\min }$ and maximum one $E_{\mathrm{BES} i}^{\max }$ by constraint (11). Indicator constraints (9), with binary variable $u_{\mathrm{BES} i}^{t}$, prevent the simultaneous charging and discharging of the batteries.

In the literature, more accurate MILP models of the BES are described (e.g., in [5], [18], [19] and [20]) that can replace the simple model represented by (7)-(11).

Both indicator constraints (4) and (9), with the associated binary variables $u_{\mathrm{i}}^{t}$ and $u_{\mathrm{BES} i}^{t}$, are used to improve the convergence and to obtain the most easily applicable solution, but do not increase the value of the objective function. Indeed, in the considered model, there is no prosumer who can benefit from the purchase and resale of energy in the same time interval, since no prosumer has restrictions to buy directly from those who sell at the lowest price. Furthermore, a simultaneous charging and discharging of the battery would have the sole effect of increasing the losses due to $\eta_{\mathrm{ch} i}$ and $\eta_{\mathrm{dis} i}$.

Due to the cooperative behavior of the prosumers, each producer tends to fulfill as much as possible the needs of nearby consumers in the network with radial configuration, so to minimize the losses. In this context, the presence of counterflows, i.e., transaction in opposite direction in the same branch, is not reasonable. This justifies the approximate evaluation of the power losses carried out separately for each prosumer in the first stage, to preserve the uncoupled structure of the problem needed by the ADMM approach. The evaluation of the losses is refined in the second stage after the losses allocation.

According to this approach, $L_{b, i}^{t}$ in (3) is defined by the following constraints

$$
\begin{aligned}
& L_{b, i}^{t}=\frac{R_{b}}{3 V_{n}^{2}}\left(F_{b, i}^{t}\right)^{2} \quad t \in T, b \in B, i \in \Omega \\
& F_{b, i}^{t}=A_{\text {Grid } b, i} P_{\text {buy_Grid } i}^{t}-A_{\text {Grid } b, i} P_{\text {sell_Grid } i}^{t} \\
& +\sum_{j \in \Omega} A_{b, i, j} P_{\text {buy } i, j}^{t}-\sum_{j \in \Omega} A_{b, i, j} P_{\text {sell } i, j}^{t} \quad t \in T, b \in B, i \in \Omega
\end{aligned}
$$


In (12), $R_{b}$ is the resistance of branch $b, V_{n}$ is the line-to-line rated voltage value and $F_{b, i}^{t}$ is the three-phase power flow in branch $b$, only due to the transaction that involves $i$, considered to be positive when directed from the substation to the end of the feeder. Constraint (12) assumes that the rms bus voltage values are equal to the rated value, reactive power flows are neglected, and the LV network is balanced.

In (13), $A_{\text {Grid }}$ and $A$ are a 2-D matrix and a 3-D array, respectively, that describe the position of each branch with respect to the buses where the prosumers are connected, assuming a radial configuration:

- $A_{\text {Grid } b, i}$ is the $b, i$ element of matrix $A_{\text {Grid }}$. It is equal to 0 if branch $b$ cannot be crossed by a power flow due to the transaction between prosumer $i$ and the external network, whilst it is 1 otherwise.

- $A_{b, i, j}$ is the $b, i, j$ element of array $A$. It is equal to 1 if branch $b$ is crossed in the assumed positive direction by the power flow created when $i$ buys from $j$, it is -1 if branch $b$ is crossed in the negative direction by the flow created when $i$ buys from $j$, and it is 0 if branch $b$ is not crossed by the flow created when $i$ buys from $j$.

In the implemented MILP model, constraint (12) is replaced by a its piecewise linear approximation described in, e.g., [21]. A set $L$ of segments is created for the linearization. Each segment is defined by breakpoints $H_{\text {Flow } b, l}^{t}$, obtained by dividing the allowed range of $F_{b}^{t}$ in $|L|$ intervals. Each breakpoint $H_{\text {Flow } b, l}^{t}$ defines a breakpoint $H_{\text {Loss } b, l}^{t}$ of the piecewise representation of $L_{b}^{t}$, which is given by

$$
\begin{aligned}
F_{b}^{t}= & \sum_{l \in L} a_{b, l}^{t} H_{\text {Flow } b, l}^{t} \quad t \in T, b \in B \\
L_{b}^{t}= & \sum_{l \in L} a_{b, l}^{t} H_{\text {Loss } b, l}^{t} \quad t \in T, b \in B \\
& \sum_{l \in L} a_{b, l}^{t}=1 \quad t \in T, l \in L
\end{aligned}
$$

where $a_{b, l}^{t}$ are SOS2 variables, i.e., they are linked with a special ordered set of type 2 constraints so that at most two and consecutive variables can be non-zero. Since in the first stage the losses are calculated separately for each transaction, the model (14)-(16) is applied for each prosumer $i$ by using power flow $F_{b, i}^{t}$ defined by (13).

\section{B. Second stage}

The second stage refines the estimation of the power loss in the internal network by considering the concurrent presence of the flows of all the transactions in each branch. Moreover, the second stage allocates the losses to each transaction.

By using the results of the first stage flow $F_{b}^{t}$ in each branch due to all the transactions is calculated as

$$
\begin{aligned}
F_{b}^{t}= & \sum_{i \in \Omega} A_{\text {Grid } b, i} P_{\text {buy_Grid } i}^{t}- \\
& \sum_{i \in \Omega} A_{\text {Grid } b, i} P_{\text {sell_Grid } i}^{t}+\sum_{i \in \Omega} \sum_{j \in \Omega} A_{b, i, j} P_{\text {buy } i, j}^{t}
\end{aligned}
$$

The total value of power loss $L_{b}^{t}$ in branch $b$ at time $t$ is calculated by replacing $F_{b, i}^{t}$ with $F_{b}^{t}$ in (12). Following a typical approach [22], the losses in each branch $b$ are proportionally attributed to the transactions that create flows in branch $b$ by using coefficients $K_{\text {buy_Grid } b, i}^{t}, K_{\text {sell_Grid } b, i}^{t}, K_{\text {buy } b, i, j}^{t}$ (if $F_{b}^{t} \neq 0$ ):

$$
\begin{aligned}
K_{\text {buy_Grid } b, i}^{t} & =A_{\text {Grid } b, i} P_{\text {buy_Grid } i}^{t} / F_{b}^{t} \\
K_{\text {sell_Grid } b, i}^{t} & =-A_{\text {Grid } b, i} P_{\text {sell_Grid } i}^{t} / F_{b}^{t} \\
K_{\text {buy } b, i, j}^{t} & =A_{b, i, j} P_{\text {buy } i, j}^{t} / F_{b}^{t} .
\end{aligned}
$$

The optimization is then repeated, by using a MILP model of the second stage in which constraints (3) are replaced by

$P_{\mathrm{G} i}^{t}+P_{\mathrm{BES} \_ \text {dis } i}^{t}+P_{\text {buy_Grid } i}^{t}-\sum_{b \in B} L_{\text {buy_Grid } b, i}^{t}+\sum_{\substack{j \in \Omega \\ j \neq i}} P_{\text {buy } i, j}^{t}=P_{\mathrm{D} i}^{t}+$

$P_{\text {BES_ch }, i}^{t}+P_{\text {sell_Grid } i}^{t}+\sum_{b \in B} L_{\text {sell_Grid } b, i}^{t}+\sum_{\substack{j \in \Omega \\ j \neq i}} P_{\text {sell } i, j}^{t}+\sum_{\substack{j \in \Omega \\ j \neq i}} \sum_{b \in B} L_{\text {buy } b, j, i}^{t}$

$$
t \in T, i \in \Omega
$$

where $L_{\text {buy_Grid } b, i}^{t}, \quad L_{\text {sell_Grid } b, i}^{t}$, and $L_{\text {buy } b, j, i}^{t}$ are the losses in branch $b$ attributed to the power bought by $i$ from the utility grid, to the power sold by $i$ to the utility grid, and to the power sold by $i$ to $j$, respectively. The losses attributed to each transaction are obtained by the product of the corresponding coefficient, given by (18), (19), and (20), and the total value of power loss $L_{b}^{t}$ in branch $b$ :

$$
\begin{gathered}
L_{\text {buy_Grid } b, i}^{t}=K_{\text {buy_Grid } b, i}^{t} L_{b}^{t} \quad t \in T, i \in \Omega, b \in B \\
L_{\text {sell_Grid } b, i}^{t}=K_{\text {sel__Grid } b, i}^{t} L_{b}^{t} \quad t \in T, i \in \Omega, b \in B \\
L_{\text {buy } b, i, j}^{t}=K_{\text {buy } b, i, j}^{t} L_{b}^{t} \quad t \in T \quad t \in T, i \in \Omega, b \in B .
\end{gathered}
$$

According to (21), each prosumer $i$ compensates for losses due to its transactions with the utility grid and sale transactions with other prosumers. In fact, we assume that both $P_{\text {buy } i, j}^{t}$ and $P_{\text {sell } i, j}^{t}$ (which corresponds to $P_{\text {buy } j, i}^{t}$ ) are measured at the buyer's connection.

Constraints (17) are included in the second stage model of the centralized approach for each branch $b$ and time interval $t$ and the corresponding power loss, namely $L_{b}^{t}$, is represented by using the piecewise linear approximation of (12) given by (14) $-(16)$.

In addition to the constraints of the first stage, the model of the second stage includes constraints that avoid transactions not present in the first stage solution:

$$
\begin{cases}P_{\text {buy_Grid } i}^{t} T_{\text {buy_Grid } i}^{t}=0 & t \in T, i \in \Omega \\ P_{\text {sell_Grid } i}^{t} T_{\text {sell_Grid } i}^{t}=0 & t \in T, i \in \Omega \\ P_{\text {buy } i, j}^{t} T_{\text {buy } i, j}^{t}=0 & t \in T, i \text { and } j \in \Omega, i \neq j\end{cases}
$$

where parameters $T_{\text {buy_Grid } i}^{t}, T_{\text {sell_Grid } i}^{t}$, and $T_{\text {buy } i, j}^{t}$ are equal to 0 if in the first stage solution $i$ buys from the utility grid, $i$ sells to the utility grid, and $i$ buys from $j$, respectively; otherwise, these parameters are equal to 1 , under the assumption that transaction decisions are not significantly affected by the losses. 


\section{PROBlem Formulation - Distributed APPROACH}

As mentioned, the distributed approach is based on the ADMM. The optimization is performed in an iterative way. At each ADMM iteration, the power bought or sold by each prosumer $j$ calculated in the previous iteration is made known to all prosumers. These values are considered as parameters in the optimization problem solved by prosumer $k$ at the current iteration and they are denoted by a hat in the model described in this section.

\section{A. First stage}

The objective function to minimize by prosumer $k$ is

$O F_{k}=\min _{\substack{P_{\text {byy_Grid }}^{t}, P_{\text {sell_Gridk }}^{t} \\ P_{\text {byy } k, j}^{t}, P_{\text {sell } k, j}^{t}}} \sum_{\substack{t \in T \\ \text { buy }}}\left[\begin{array}{l}\pi_{\text {buy }}^{t} P_{\text {buy_Grid } k}^{t} \Delta t-\pi_{\text {sell }}^{t} P_{\text {sell_Grid } k}^{t} \Delta t+ \\ \sum_{\substack{j \in \Omega \\ j \neq k}} \lambda_{j}^{t} P_{\text {buy } k, j}^{t} \Delta t-\lambda_{k}^{t} \sum_{\substack{j \in \Omega \\ j \neq k}} P_{\text {sell } k, j}^{t} \Delta t+\ell_{k}^{t}\end{array}\right]$

where

$$
\ell_{k}^{t}=m \cdot \rho \cdot\left[\sum_{\substack{j \in \Omega \\ j \neq k}}\left(\hat{P}_{\text {buy } j, k}^{t}-P_{\text {sell } k, j}^{t}\right)^{2}+\sum_{\substack{j \in \Omega \\ j \neq k}}\left(P_{\text {buy } k, j}^{t}-\hat{P}_{\text {sell } j, k}^{t}\right)^{2}\right]
$$

Equation (26) is obtained by the decomposition of the Lagrangian for each prosumer $k$. The Lagrangian incorporates $O F$ (1) and constraints (2), each multiplied by the relevant Lagrange multiplier $\lambda_{i}^{t}$. According to the ADMM, the Lagrangian is augmented by the squared norm of the same constraints (2) multiplied by positive penalty parameter $\rho$ and fixed scale factor $m$, as shown in (27).

$O F_{k}$ can be seen as the summation of the costs of the energy purchased by $k$ from the utility grid at price $\pi_{\text {buy }}^{t}$ and from each other prosumer $j$ at price $\lambda_{j}^{t}$, minus the sum of revenues due to the energy sold by $k$ to the utility grid at price $\pi_{\text {sell }}^{t}$ and to the other prosumers at price $\lambda_{k}^{t}$. Once the procedure converges, $\ell_{k}^{t}$ is zero and the value $O F$ for the entire system is the sum of the ones of each prosumer $k$ :

$$
O F=\sum_{k \in \Omega} O F_{k}
$$

The optimization problem of prosumer $k$ includes constraints (3)-(11) and (13)-(16) for $i=k$.

Furthermore, the convergence of the ADMM procedure is improved by adding the following constraints, starting from the second iteration, as they provide a coordination between the sales and purchase decisions of prosumer $k$ with respect to those of the other prosumers:

$$
\begin{gathered}
P_{\text {sell } k, j}^{t} \leq \hat{P}_{\text {buy_Grid } j}^{t}+\sum_{\substack{i \in \Omega \\
i \neq j}} \hat{P}_{\text {buy } j, i}^{t} \quad t \in T, j \in \Omega \\
P_{\text {buy } k, j}^{t} \leq \hat{P}_{\text {sell_Grid } j}^{t}+\sum_{\substack{i \in \Omega \\
i \neq j}} \hat{P}_{\text {sell } j, i}^{t} \quad t \in T, j \in \Omega
\end{gathered}
$$

At each iteration $v$, after the solution of the optimization problem, prosumer $k$ communicates both $P_{\text {buy } k, j}^{t}$ and $P_{\text {sell } k, j}^{t}$ to every other prosumer $j$. When all the prosumers have solved their own optimization problems, they update Lagrangian multipliers $\lambda_{k}^{t}$. For this purpose, let $r_{k}^{v}$ be the primal residual term for prosumer $k$, equal to the vector with $|T|$ elements

$$
r_{k}^{t}=\sum_{\substack{j \in \Omega \\ j \neq k}} P_{\text {buy } j, k}^{t}-\sum_{\substack{j \in \Omega \\ j \neq k}} P_{\text {sell } k, j}^{t},
$$

the $|T|$-dimensional vector of Lagrangian multipliers $\lambda_{k}^{v}$, with elements $\lambda_{k}^{t}$, is updated as

$$
\lambda_{k}^{v+1}=\lambda_{k}^{v}+2 m \cdot \rho \cdot r_{k}^{v} .
$$

The procedure is iteratively repeated until the absolute value of all residuals $r_{k}^{t}$ is less than tolerance $\varepsilon$ (which is assumed to be equal to $10 \mathrm{~W}$ in all the numerical tests of this paper). At the beginning of the ADMM procedure, $\lambda_{k}^{t}$ is initialized to be equal to $1 / 2\left(\pi_{\text {buy }}^{t}+\pi_{\text {sell }}^{t}\right)$.

The penalty parameters are updated as follows [8]

$$
\rho^{v+1}= \begin{cases}2 \rho^{v} & \left\|r_{k}^{v}\right\|_{2}>10\left\|s_{k}^{v}\right\|_{2} \\ \rho^{v} / 2 & \left\|s_{k}^{v}\right\|_{2}>10\left\|r_{k}^{v}\right\|_{2} \\ \rho^{v} & \text { otherwise }\end{cases}
$$

where \|\|$_{2}$ is the Euclidian norm and $s_{k}^{v}$ is the $|T|$-dimensional vector of the dual residual elements

$$
s_{k}^{t}=\rho^{v}\left[\begin{array}{l}
\left(\sum_{j \in \Omega} P_{\text {sell } k, j}^{t}\right)^{v}-\left(\sum_{j \in \Omega} P_{\text {sell } k, j}^{t}\right)^{v-1} \\
+\left(\sum_{j \in \Omega} P_{\text {buy } k, j}^{t}\right)^{v}-\left(\sum_{j \in \Omega} P_{\text {buy } k, j}^{t}\right)^{v-1}
\end{array}\right] .
$$

To accelerate the convergence, the initial value of $m$, which is equal to $1 \cdot 10^{-5}$, is multiplied by 5 and by 1.5 when the maximum value of the total mismatch $r^{t}=\sum_{k}\left|r_{k}^{t}\right|$ becomes less than $20 \mathrm{~kW}$ and $1 \mathrm{~kW}$, respectively, and further multiplied by 5 when $\max \left(\left|r_{k}^{t}\right|\right)<100 \mathrm{~W}$.

The consensus activity required to coordinate the various prosumers may be performed by using a distributed ledger.

As described in [8], the convergence of ADMM to a global optimal point is not guaranteed when it is applied to nonconvex problems. It will possibly have better convergence than other local optimization methods and it has been successfully applied to large-scale mixed integer problems as shown in, e.g., [11]. In the models considered in this paper, the binary variables are used only in indicator constraints (4) and (9). As already described in Section II, these constraints do not affect the optimal value of the $O F$ but are useful for finding the solution, among those with the optimal value, which can be more easily applied, by avoiding prosumers who buy and sell energy at the same price without benefit. Furthermore, these constraints together with (29) and (30) make the ADMM convergence significantly faster.

\section{B. Second stage}

The internal network power loss allocation follows the same rules and criteria described for the centralized approach. 
However, in order to avoid constraint (17) in the distributed approach, which would link together the purchase and sales decisions of all the prosumers, we introduce the efficiencies of each transaction (between $k$ and the grid or between $k$ and $j$ ):

$$
\begin{gathered}
\eta_{\text {buy_Grid } k}^{t}=1-\frac{\sum_{b \in B} L_{\text {buy_Grid } b, k}^{t}}{P_{\text {buy_Grid } k}^{t}} \quad t \in T \\
\eta_{\text {sell_Grid } k}^{t}=\frac{P_{\text {sell_Grid } k}^{t}}{P_{\text {sell_Grid } k+\sum_{b \in B}^{t} L_{\text {sell_Grid } b, k}^{t}}^{t}} \quad t \in T \\
\eta_{\text {buy } k, j}^{t}=\frac{P_{\text {buy } k, j}^{t}=}{P_{\text {buy } k, j}^{t}+\sum_{b \in B} L_{\text {buy } b, k, j}^{t}} \quad t \in T, j \in \Omega, j \neq k
\end{gathered}
$$

where $L_{\text {buy_Grid } b, i}^{t}, L_{\text {sell_Grid } b, i}^{t}$, and $L_{\text {buy } b, k, j}^{t}$ are calculated at the end of the first stage by using (22)-(24), in which the $L_{b}^{t}$ values are given by replacing $F_{b, i}^{t}$ with $F_{b}^{t}$ in (12) and $K$ values are calculated from (18)-(20). The $\eta$ calculation can be carried out by a central coordinator that knows the topology and parameters of the network and collects the power exchanges of the first stage (i.e. the values $P_{\text {buy } k, j}^{t}, P_{\text {buy_Grid k }}^{t}, P_{\text {sell_Gridk }}^{t}$ from each prosumer $k$ ). This calculation can be also done by each prosumer in a distributed manner (assuming that each prosumer knows resistance values of the branches and matrices $A_{\text {Grid b,i }}$, $A_{b, i, j}$ ) provided that each prosumer $k$ communicates its power exchanges to every other prosumer of the LEC.

These efficiency parameters are included in the balance constraint of each prosumer $k$. Then constraints (21) become:

$$
\begin{array}{r}
P_{\mathrm{G} k}^{t}+P_{\mathrm{BES} \_ \text {dis } k}^{t}+\eta_{\text {buy_Grid } k}^{t} P_{\text {buy_Grid } k}^{t}+\sum_{\substack{j \in \Omega \\
j \neq k}} P_{\text {buy } k, j}^{t}= \\
P_{\mathrm{D} k}^{t}+P_{\mathrm{BES} \_ \text {ch } k}^{t}+\frac{P_{\text {sell_Grid } k}^{t}}{\eta_{\text {sell_Grid } k}^{t}}+\sum_{\substack{j \in \Omega \\
j \neq k}} \frac{P_{\text {sell } k, j}^{t}}{\eta_{\text {buy } j, k}} \\
t \in T, k \in \Omega
\end{array}
$$

As the centralized model, the second stage models of the ADMM procedure include also constraints (25) for $k=i$.

For a complete comparison of the ADMM procedure with the centralized approach, a second stage model of the centralized approach was implemented in which (38) replaces (21). In Section IV, we denote this model as centralized- $\eta$.

\section{IMPLEMENTATION AND TEST RESULTS}

The models have been implemented in the AIMMS Developer modelling environment [23] and tested by using the Cplex V12.8 solver [24] on a 2-GHz Intel-i7 computer with 8 GB of RAM, running 64-bit Windows 10. The MILP solver is used for the centralized model and the MIQP (mixed integer quadratic programming) solver for the ADMM model.

All the calculations refer to a time window of 1 day, divided into 96 periods of 15 minutes each.

The test system is composed of two LV feeders. Each feeder consists of five lines, each with resistance $R_{b}=189 \mathrm{~m} \Omega$. Five prosumers are connected to each feeder: prosumers 1-5 to a feeder and prosumers 6-10 to the other. Each prosumer is equipped with a PV system and a load. We repeat the calculations two times: once without BES units and the other by assuming that each prosumer is also equipped with a BES unit. The total daily consumption of the LEC is $313 \mathrm{kWh}$ and the corresponding PV production is $231 \mathrm{kWh}(73.8 \%$ of the load). The load profiles adopted for each prosumer are shown in Fig. 2. For all the PV units we assumed the same profile of the ratio between power output and panel surface shown in Fig. 3. The area of the PV panel of each prosumer is given in Table I. Fig. 3 also shows the price profile of the energy bought from the utility grid $\pi_{\text {buy }}^{t}$. We assume that the price of the energy sold by the LEC to the utility grid (i.e., $\pi_{\text {sell }}^{t}$ ) is half of $\pi_{\text {buy }}^{t}$. Sizes $E_{\mathrm{BES}}^{\max }$ of the BES units are shown in Table II and the corresponding $P_{\mathrm{BES}}^{\max }$ values are assumed to be equal to the ratio $E_{\mathrm{BES}}^{\max } / \Delta t$. The total capacity of the BES units is $30 \mathrm{kWh}(13 \%$ of the daily $\mathrm{PV}$ production).

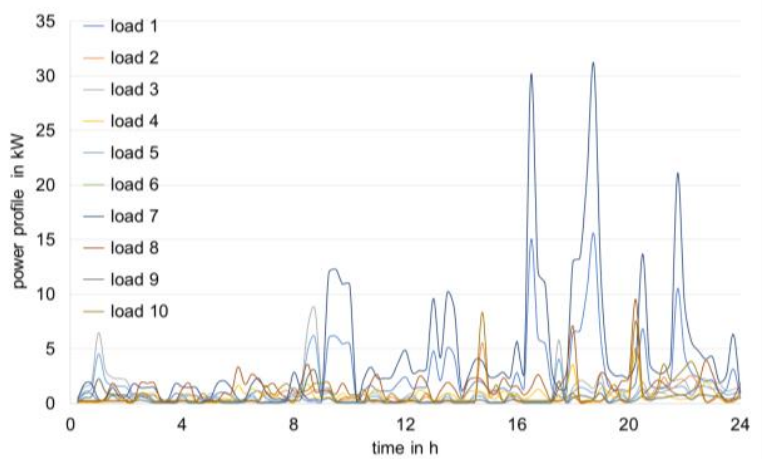

Fig. 2. Load profile for each prosumer.

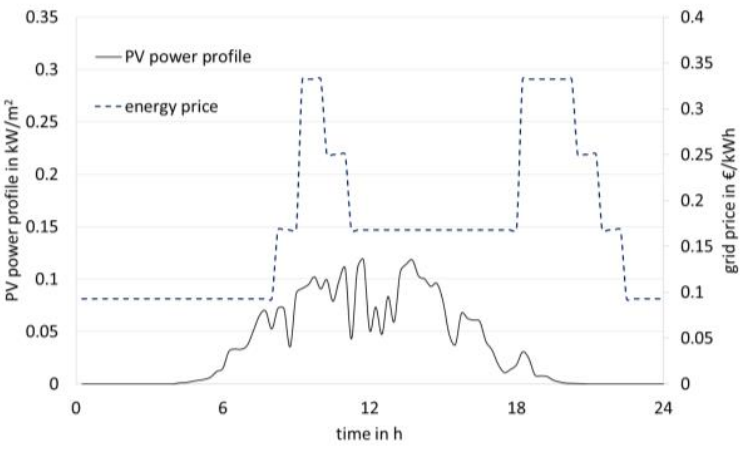

Fig. 3. Profile of the PV production and grid purchase price.

TABLE I - PV PANEL SURFACE FOR EACH PROSUMER

\begin{tabular}{c|c|c|c|c|c|c|c|c|c|c}
\hline \hline prosumer & 1 & 2 & 3 & 4 & 5 & 6 & 7 & 8 & 9 & 10 \\
\hline area $\left(\mathrm{m}^{2}\right)$ & 32 & 14 & 21 & 32 & 28 & 14 & 42 & 32 & 14 & 42 \\
\hline \hline & TABLE II - SIZES OF THE BES UNITS \\
\hline \hline prosumer & 1 & 2 & 3 & 4 & 5 & 6 & 7 & 8 & 9 & 10 \\
\hline size $(\mathrm{kWh})$ & 5 & 3 & 4 & 2 & 3 & 1 & 2 & 2 & 2 & 6 \\
\hline
\end{tabular}

\section{A. Scenario 1: prosumers without batteries}

Table III shows the $O F$ values for both stages and the total network losses in the 24 hours, for the centralized model, the centralized- $\eta$ model and the distributed approach without BES units. The results are similar.

If the optimization problems of the prosumers are solved in sequence, without considering delays or limitations in the communication channels, the distributed model requires a 
solution time around $60 \mathrm{~s} / 13$ iterations for stage 1 and $80 \mathrm{~s} / 8$ iterations for stage 2 . The solution of the centralized model needs around $1 \mathrm{~s}$ for each stage. As expected, the computational effort decreases if a longer $\Delta t$ is adopted. For example, if $\Delta t=30$ min, the solution of the centralized first stage model needs around $0.2 \mathrm{~s}$, whilst the distributed model requires $25 \mathrm{~s}$. If $\Delta t=1$ $\mathrm{h}$, the centralized first stage model needs around $0.1 \mathrm{~s}$, whilst the distributed model requires $14 \mathrm{~s}$. As mentioned in the Introduction, the purpose of adopting the distributed approach instead of the centralized one is not to improve the computational performance but to reduce the amount of shared information.

\begin{tabular}{|c|c|c|c|}
\hline & \multicolumn{2}{|c|}{ OF $(€)$} & \multirow{2}{*}{$\begin{array}{c}\text { Losses (kWh) } \\
\text { stage } 2 \\
\end{array}$} \\
\hline & stage 1 & stage 2 & \\
\hline Centralized & 26.85 & 27.19 & 3.65 \\
\hline Centralized- $\eta$ & 26.85 & 27.18 & 3.62 \\
\hline ADMM & 26.87 & 27.18 & 3.63 \\
\hline
\end{tabular}

The reasonable accuracy of the power loss representation in the second stage of both the centralized and distributed optimization models is confirmed by the maximum percentage difference of a few percent between the power loss calculated at the end of stage 2 for each period and the corresponding values obtained by (12) with $F_{b, i}^{t}$ replaced by $F_{b}^{t}$.

To illustrate the convergence behavior of the ADMM procedure, Fig. 4 shows the average value of the primal residuals $r_{k}^{t}$ (denoted by $R$ ) at each iteration, the corresponding value of the augmented $O F$ according to (28), and the $O F$ value of (1).

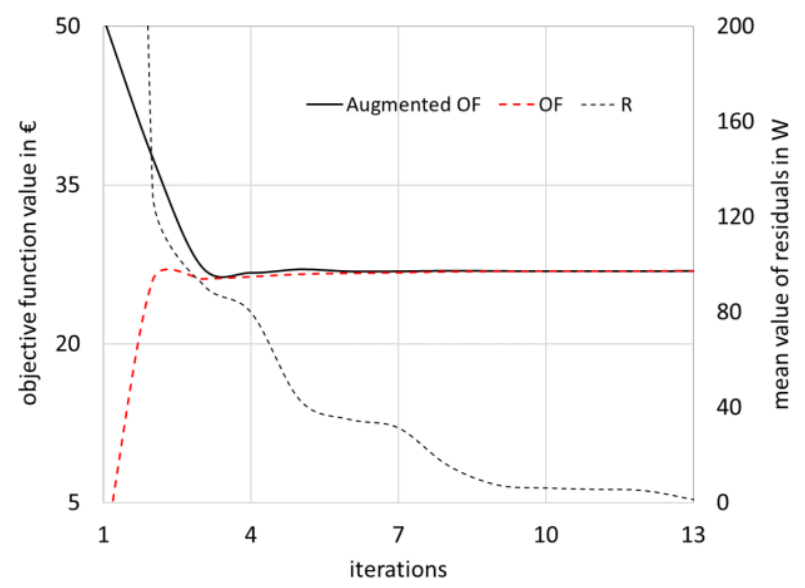

Fig. 4. ADMM convergence in stage 1 for scenario 1: average value $R$ of the primal residuals at each iteration, augmented $O F, O F$ value corresponding to the exchanges with the utility grid.

As shown in Fig. 5 the profiles of the power exchanged by the LEC with the utility grid calculated by using the two approaches are similar.

Fig. 6 shows the profiles of the power flow from each prosumer when it sells to the others calculated by using the centralized model and the ADMM approach.

In Fig. 7 the colored dotted lines correspond to the prices of the energy purchased and sold to the utility grid (i.e., $\pi_{\text {buy }}^{t}$ and $\left.\pi_{\text {sell }}^{t}\right)$, whilst the black dots represent the prices of prosumers when they sell energy to any other prosumer of the LEC. For the case of the centralized model, the prices correspond to the Lagrangian multiplier associated to (2). The comparison between Fig. 7a) and Fig. 5 shows that the prices are equal to $\pi_{\text {buy }}^{t}$ when the LEC, as a whole, imports energy from the utility grid and they are equal to $\pi_{\text {sell }}^{t}$ when the LEC sells energy to the utility grid. Analogous results are obtained by using the ADMM procedure shown in Fig. 7b). In this case the prices are the values given by (32) at the last iteration.

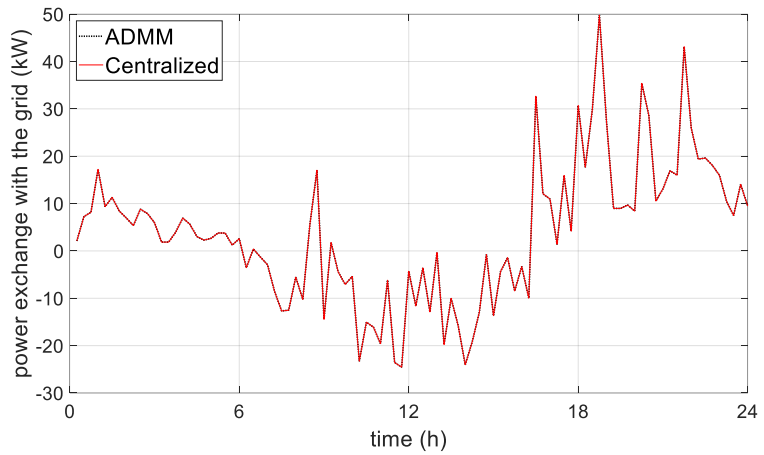

Fig. 5. Comparison of the power flow at the connection with the utility grid (positive if consumed by the LEC) (solid line: centralized model, dashed line: ADMM model).
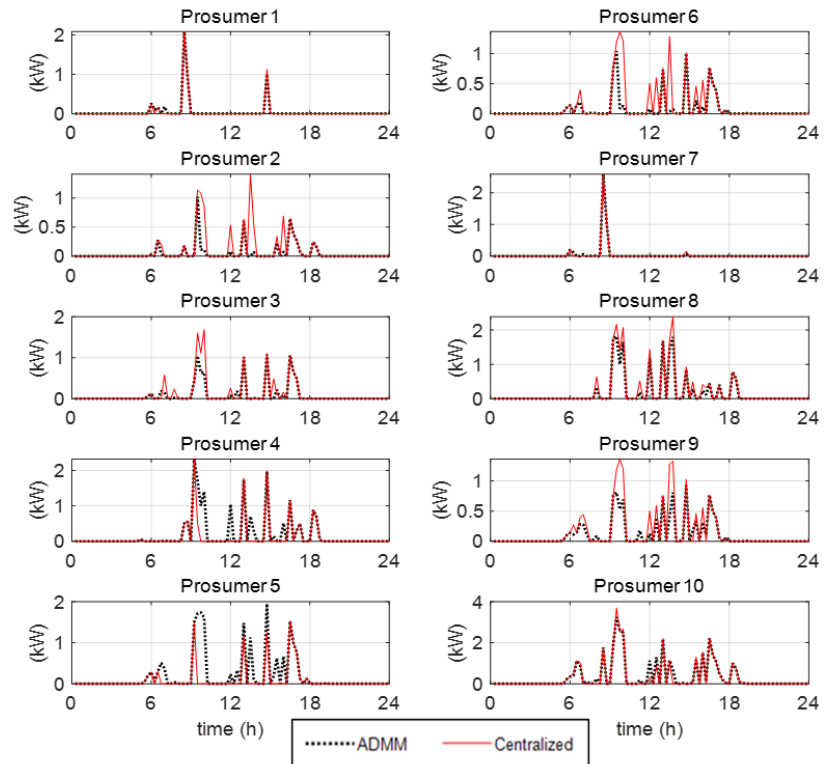

Fig. 6. Comparison of the power flows from every prosumer when it sells to the others (excluding the utility grid) for scenario 1 (solid line: centralized model, dashed line: ADMM model).

Table IV and Table $\mathrm{V}$ show the individual energy procurement costs of each prosumer for the centralized and the ADMM approaches, taking into account both the exchanges with the external grid and the internal exchanges and the prices of Fig. 7. Clearly, the effect of the internal exchanges on the total $O F$ value given by (1) and (28) is compensated, i.e., the sum of the individual costs of Table IV and Table $\mathrm{V}$ is equal to the corresponding $O F$ values reported in Table III.

Moreover, Table IV and Table V show the individual cost/revenue values obtained by assuming that each prosumer can only transact with the external energy provider, preventing 
exchanges with other prosumers (i.e., $P_{\text {buy } i, j}^{t}$ and $P_{\text {sell } j, i}^{t}$ are tied to zero). The comparison of these results with those obtained by both the centralized and the ADMM procedures shows that each single prosumer has a lower cost or an improved revenue inside the LEC with respect to the case in which it exchanges only with the external provider. As expected, also the total procurement energy cost of the LEC is reduced (of about 11\%) with respect to the total cost of the same set of prosumers when they operate without mutual exchanges.

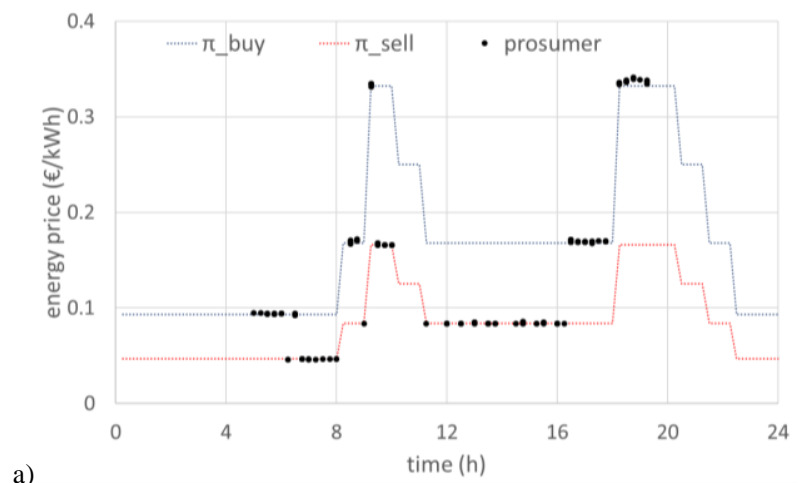

a)

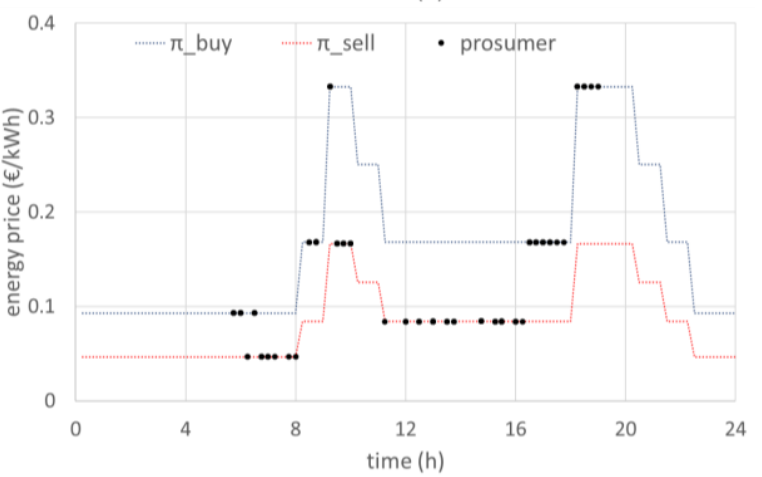

Fig. 7. Energy prices of the selling prosumers for scenario 1: a) centralized model, b) last iteration of the ADMM procedure.

TABLE IV - ENERGy PROCUREMENT Cost IN $€$ (NEGATIVE VALUES INDICATE REVENUES) FOR EACH PROSUMER IN FEEDER 1 WITHOUT BES UNITS

\begin{tabular}{c|c|c|c|c|c}
\hline \hline prosumer & 1 & 2 & 3 & 4 & 5 \\
\hline Centralized & 6.81 & 0.87 & 2.09 & -0.43 & 0.23 \\
\hline Centralized- $\eta$ & 6.82 & 0.87 & 2.09 & -0.43 & 0.22 \\
\hline ADMM & 6.83 & 0.89 & 2.08 & -0.44 & 0.19 \\
\hline $\begin{array}{c}\text { without internal } \\
\text { exchanges }\end{array}$ & 7.36 & 1.03 & 2.16 & -0.20 & 0.37 \\
\hline \hline
\end{tabular}

TABLE V - ENERGy PROCUREMENT COST IN $€$ (NEGATIVE VALUES INDICATE REVENUES) FOR EACH PROSUMER IN FEEDER 2 WITHOUT BES UNITS

\begin{tabular}{c|c|c|c|c|c}
\hline \hline prosumer & 6 & 7 & 8 & 9 & 10 \\
\hline Centralized & 0.08 & 15.90 & 2.17 & 0.10 & -0.65 \\
\hline Centralized- $\eta$ & 0.08 & 15.90 & 2.17 & 0.10 & -0.65 \\
\hline ADMM & 0.08 & 15.95 & 2.17 & 0.09 & -0.66 \\
\hline $\begin{array}{c}\text { without internal } \\
\text { exchanges }\end{array}$ & 0.16 & 17.45 & 2.40 & 0.18 & -0.22 \\
\hline \hline
\end{tabular}

B. Scenario 2: prosumers with batteries

Table VI compares the $O F$ values for both stages and the total network losses in the 24 hours obtained by including the BES units in the models. The distributed model solution time is around $300 \mathrm{~s} / 46$ iterations for stage 1 and $180 \mathrm{~s} / 82$ iterations for stage 2 . The solution of the centralized model needs around
$10 \mathrm{~s}$ for stage 1 and around $8 \mathrm{~s}$ for stage 2 .

TABLE VI - COMPARISON BETWEEN CENTRALIZED MODEL AND ADMM SCENARIO 2

\begin{tabular}{c|c|c|c}
\hline \hline & \multicolumn{2}{|c|}{ OF $(€)$} & Losses (kWh) \\
& stage 1 & stage 2 & stage 2 \\
\hline Centralized & 18.06 & 18.35 & 3.41 \\
\hline Centralized- $\eta$ & 18.06 & 18.36 & 3.35 \\
\hline ADMM & 18.12 & 18.39 & 3.44 \\
\hline \hline
\end{tabular}

The convergence performance of the ADMM procedure is illustrated in Fig. 8.

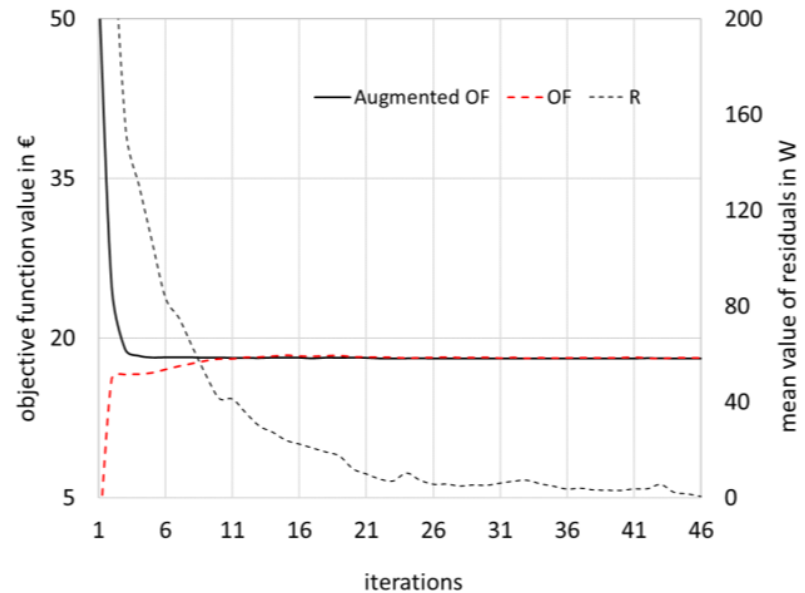

Fig. 8. ADMM convergence in stage 1 for scenario 2: average value $R$ of primal residuals at each iteration, augmented $O F, O F$ value corresponding to the exchanges with the utility grid.

The total power flow at the connection of the LEC with the utility grid and the power profiles from each prosumer when it sells to the others are compared in Fig. 9 and Fig. 10, respectively.

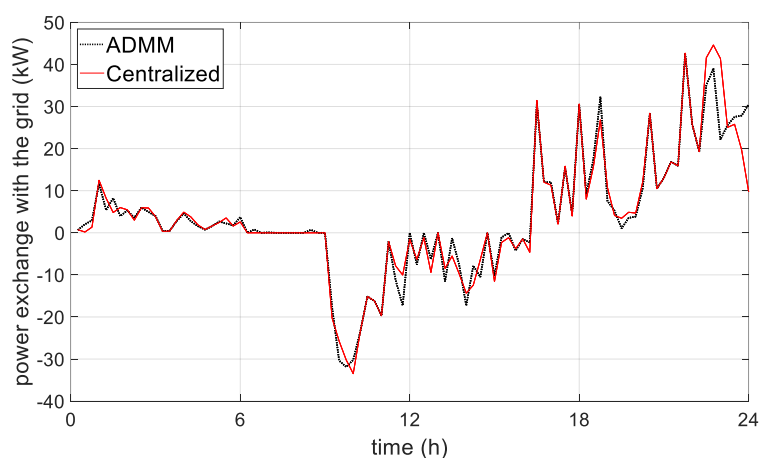

Fig. 9. Comparison of the power flow exchanged with the utility grid (positive if consumed by the LEC) for scenario 2 (solid line: centralized model, dashed line: ADMM model).

Fig. 11 compares the profiles of the total energy contained in the BES units of the LEC, whilst Fig. 12 provides the detail of the SoE profiles of each BES unit. 

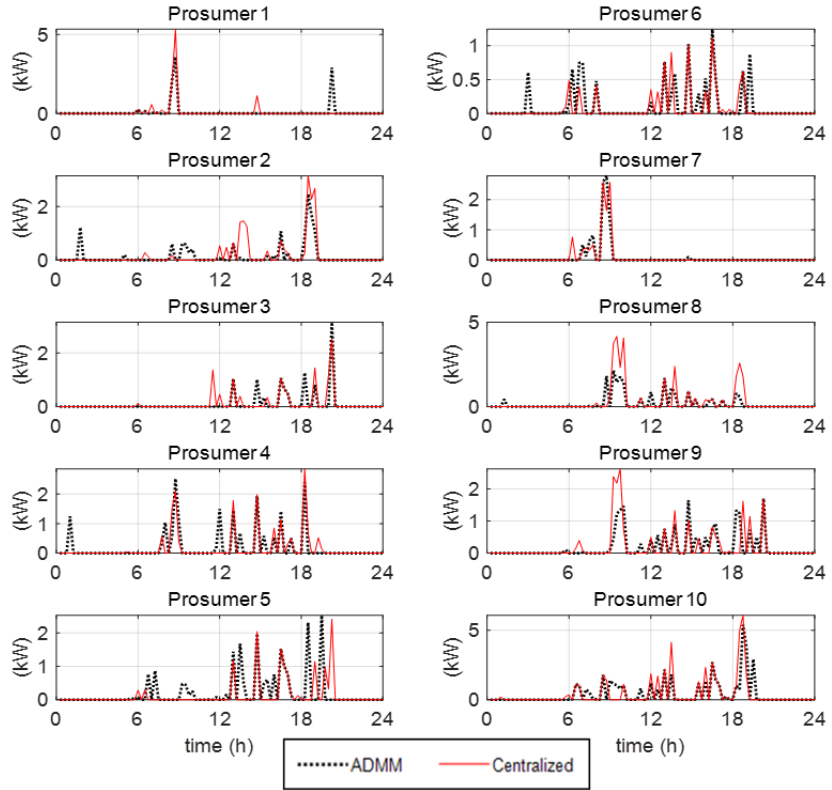

Fig. 10. Comparison of the power flow from every prosumer when it sells to the others (excluding the utility grid) for scenario 2 (solid line: centralized model, dashed line: ADMM model).

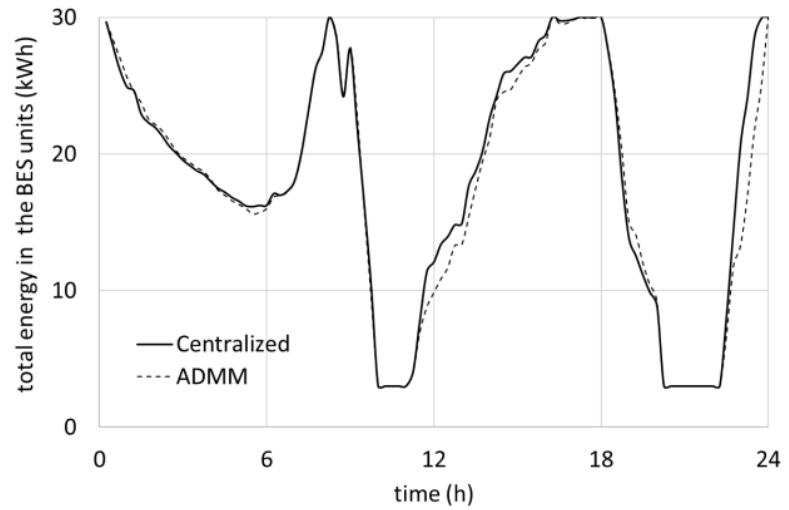

Fig. 11. Comparison of the total energy in the batteries of the LEC obtained by the centralized and the ADMM approach.

TABLE VII - ENERGY PROCUREMENT COST IN $€$ (NEGATIVE VALUES INDICATE REVENUES) FOR EACH PROSUMER IN FEEDER 1 WITH BES UNITS

\begin{tabular}{c|c|c|c|c|c}
\hline \hline prosumer & 1 & 2 & 3 & 4 & 5 \\
\hline Centralized & 5.25 & 0.08 & 0.97 & -0.96 & -0.62 \\
\hline Centralized- $\eta$ & 5.25 & 0.09 & 0.98 & -0.97 & -0.63 \\
\hline ADMM & 5.26 & 0.10 & 0.99 & -0.96 & -0.61 \\
\hline $\begin{array}{c}\text { without internal } \\
\text { exchanges }\end{array}$ & 5.47 & 0.29 & 1.12 & -0.79 & -0.43 \\
\hline \hline
\end{tabular}

TABLE VIII - ENERGY PROCUREMENT COST IN $€$ (NEGATIVE VALUES INDICATE REVENUES) FOR EACH PROSUMER IN FEEDER 2 WITH BES UNITS

\begin{tabular}{c|c|c|c|c|c}
\hline \hline prosumer & 6 & 7 & 8 & 9 & 10 \\
\hline Centralized & -0.21 & 14.85 & 1.67 & -0.45 & -2.25 \\
\hline Centralized- $\eta$ & -0.21 & 14.86 & 1.66 & -0.45 & -2.23 \\
\hline ADMM & -0.20 & 14.85 & 1.66 & -0.44 & -2.24 \\
\hline $\begin{array}{c}\text { without internal } \\
\text { exchanges }\end{array}$ & -0.14 & 16.47 & 1.74 & -0.29 & -1.63 \\
\hline \hline
\end{tabular}

Fig. 13 shows the energy prices $\lambda_{i}^{t}$ of each prosumer $i$. As in Fig. 7 the colored dotted lines correspond to the prices of the energy bought from and sold to the utility grid (i.e., $\pi_{\text {buy }}^{t}$ and $\pi_{\text {sell }}^{t}$
), while the black dots represent the transaction prices of the various prosumers when they sell energy to any other prosumer of the LEC. The comparison between Fig. 13 and Fig. 9 shows that the prices of the internal transactions are not equal to $\pi_{\text {buy }}^{t}$ or $\pi_{\text {sell }}^{t}$ only during the time interval (starting just after $6 \mathrm{am}$ ) when there is no power exchange with the utility grid.
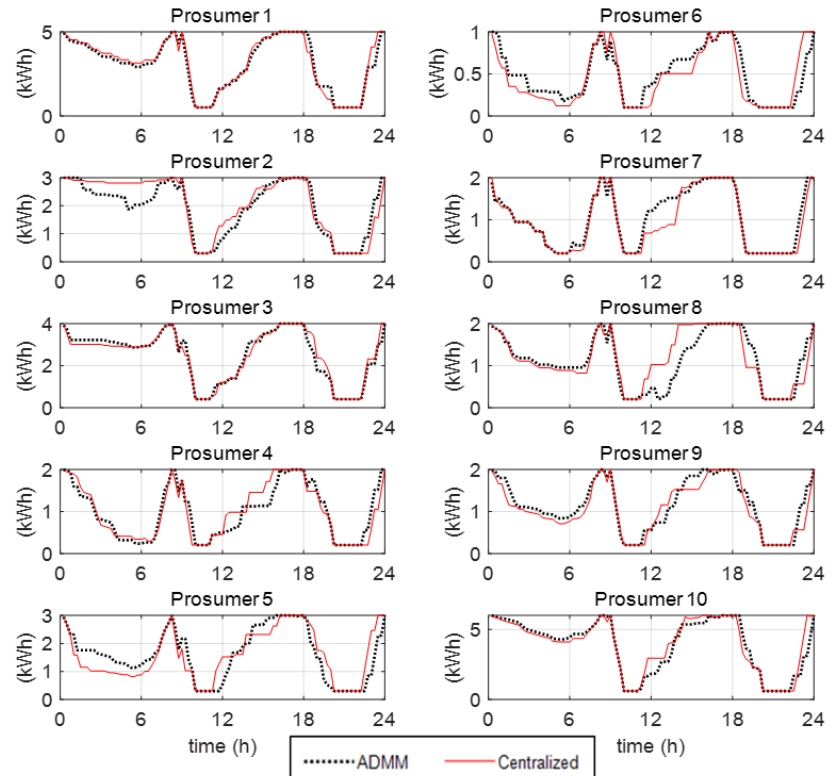

Fig. 12. Battery $S o E$ for each prosumer for scenario 2 (solid line: centralized model, dashed line: ADMM model).

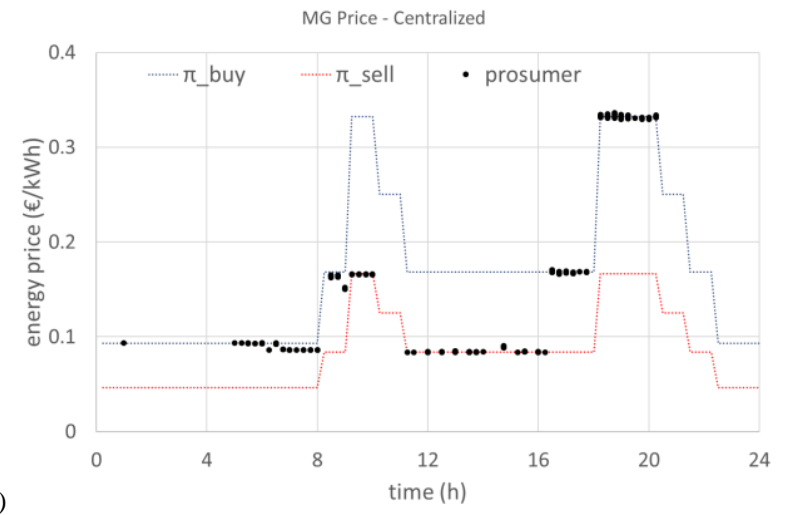

a)

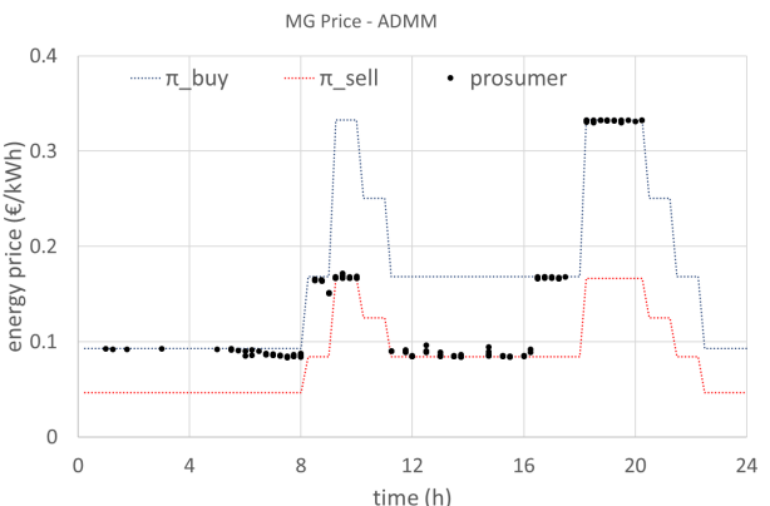

b)

Fig. 13. Energy prices of selling prosumers for scenario 2: a) centralized model, b) last iteration of the ADMM procedure.

Table VII and Table VIII compares the individual energy procurement costs of each prosumers, taking into account both 
the exchanges with the external grid and the internal exchanges and the prices of Fig. 13. Additionally, the tables show the corresponding values obtained by preventing the transactions between prosumers.

Despite the differences in the individual profiles shown in Fig. 10 and Fig. 12, the comparison presented in Table VII and Table VIII shows a reasonable match between the results given by the centralized and distributed approaches. The total energy procurement cost of the LEC is around 16\% less than the corresponding cost without internal transaction among the prosumers.

\section{CONCLUSIONS}

The proposed ADMM-based distributed optimization procedure for the day-ahead scheduling of a local energy community with generation, loads and battery storage systems incorporates the calculation of the losses and their allocation to each transaction.

A centralized approach implies that the prosumers communicate all the details of the equipment features as well as the load and production forecasts. The distributed procedure reduces the amount of shared information: in fact, the only information that every prosumer must communicate is the profile of the exchanges with the external grid and with the other prosumers for updating the multipliers at each iteration and for the evaluation of the transaction efficiencies.

The results obtained by using the distributed procedure have been compared with those of the centralized approach based on a mixed integer linear programming model. Both centralized and distributed procedures give comparable results with an acceptable computation effort. The values of the objective function, the profiles of the power flow at the connection with the utility grid and the profiles of the total energy stored in the batteries match.

The structure of the day-ahead scheduling procedures is consistent with the billing scheme and the meters of the LEC. Despite the different methods used to calculate the prices of the internal transactions in the centralized model and in the distributed procedure, the price profiles obtained by using the two approaches are similar for both the considered scenarios, i.e., with and without the presence of BES units.

The results confirm that, in the considered LEC framework, each prosumer achieves a reduction in costs or increases revenues by participating in the LEC compared to the case in which it can only transact with an external energy provider.

\section{ACKNOWLEDGMENT}

The authors thank Beatriz Ildefonso for her help in the model implementation. Special thanks to Prof. Carlo Alberto Nucci for helpful discussions during the development of this work.

\section{REFERENCES}

[1] J. Lee, J. Guo, J. K. Choi, and M. Zukerman, "Distributed energy trading in microgrids: A game-theoretic model and its equilibrium analysis," IEEE Trans. Ind. Electron., vol. 62, no. 6, pp. 3524-3533, 2015.

[2] H. Le Cadre, P. Jacquot, C. Wan, and C. Alasseur, "Peer-to-Peer Electricity Market Analysis: From Variational to Generalized Nash Equilibrium,"
arXiv:1812.02301 [math.OC], 2018.

[3] E. Mengelkamp, J. Gärttner, K. Rock, S. Kessler, L. Orsini, and C. Weinhardt, "Designing microgrid energy markets: A case study: The Brooklyn Microgrid," Appl. Energy, vol. 210, pp. 870-880, 2018.

[4] B. Prasad, E. Koliou, J. Friege, R. A. Hakvoort, and P. M. Herder, "Energetic communities for community energy : a review of key issues and trends shaping integrated community energy systems," Renew. Sustain. Energy Rev., vol. 56, pp. 722-744, 2016.

[5] C. Orozco, A. Borghetti, S. Lilla, G. Pulazza, and F. Tossani, "Comparison between multistage stochastic optimization programming and Monte Carlo simulations for the operation of local energy systems," in IEEE International Conference on Environment and Electrical Engineering (EEEIC), 2018.

[6] E. Munsing, J. Mather, and S. Moura, "Blockchains for decentralized optimization of energy resources in microgrid networks," in 2017 IEEE Conference on Control Technology and Applications (CCTA), 2017, pp. 2164-2171.

[7] M. L. Di Silvestre, P. Gallo, M. G. Ippolito, and E. Riva Sanseverino, “A technical approach to the energy blockchain in microgrids," IEEE Trans. Ind. Informatics, vol. 14, no. 11, pp. 4792-4803, 2018.

[8] S. Boyd, N. Parikh, E. Chu, B. Peleato, and J. Eckstein, Distributed optimization and statistical learning via the alternating direction method of multipliers. Foundations and Trends in Machine Learning, 2011.

[9] A. Kargarian et al., "Toward distributed/decentralized DC optimal power flow implementation in future electric power systems," IEEE Trans. Smart Grid, vol. 9, no. 4, pp. 2574-2594, 2018.

[10] H. Wang and J. Huang, "Incentivizing energy trading for interconnected microgrids," IEEE Trans. Smart Grid, vol. 9, no. 4, pp. 2647-2657, 2018.

[11] V. Dvorkin, J. Kazempour, L. Baringo, and P. Pinson, "A ConsensusADMM Approach for Strategic Generation Investment in Electricity Markets," Proc. IEEE Conf. Decis. Control, vol. 2018-Decem, no. September, pp. 780-785, 2019.

[12] Y. Zheng, Y. Song, D. J. Hill, and Y. Zhang, "Multiagent system based microgrid energy management via asynchronous consensus ADMM," IEEE Trans. Energy Convers., vol. 33, no. 2, pp. 886-888, 2018.

[13] Y. Liu et al., "Distributed robust energy management of a multimicrogrid system in the real-time energy market," IEEE Trans. Sustain. Energy, vol. 10, no. 1, pp. 396-406, 2019.

[14] W. J. Ma, J. Wang, V. Gupta, and C. Chen, "Distributed energy management for networked microgrids using online ADMM with regret," IEEE Trans. Smart Grid, vol. 9, no. 2, pp. 847-856, 2018.

[15] Y. Zhao, J. Yu, M. Ban, Y. Liu, and Z. Li, "Privacy-preserving economic dispatch for an active distribution network with multiple networked microgrids," IEEE Access, vol. 6, pp. 38802-38819, 2018.

[16] B. Zhao et al., "Energy management of multiple-microgrids based on a system of systems architecture," IEEE Trans. Power Syst., vol. 33, no. 6, pp. 6410-6421, 2018.

[17] F. Moret and P. Pinson, "Energy Collectives: a Community and Fairness based Approach to Future Electricity Markets," IEEE Trans. Power Syst., vol. in press, pp. 1-11, 2019.

[18] A. Sakti et al., "Enhanced representations of lithium-ion batteries in power systems models and their effect on the valuation of energy arbitrage applications," J. Power Sources, vol. 342, pp. 279-291, 2017.

[19] C. Bordin, H. O. Anuta, A. Crossland, I. L. Gutierrez, C. J. Dent, and D. Vigo, "A linear programming approach for battery degradation analysis and optimization in offgrid power systems with solar energy integration," Renew. Energy, vol. 101, pp. 417-430, 2017.

[20] H. Pandzic and V. Bobanac, "An accurate charging model of battery energy storage," IEEE Trans. Power Syst., pp. 10, early access, 2018.

[21] H. P. Williams, Model building in mathematical programming, 3rd ed. John Wiley \& Sons, Chichester, 1990.

[22] A. J. Conejo, J. M. Arroyo, N. Alguacil, and A. L. Guijarro, "Transmission loss allocation: a comparison of different practical algorithms," IEEE Power Eng. Rev., vol. 22, no. 5, p. 66, 2002.

[23] M. Roelofs and J. Bisschop, AIMMS Language Reference. 2013.

[24] IBM/ILOG, Introducing IBM ILOG CPLEX Optimization Studio V12.8. 2018.

[25] S. Lilla et al., "Mixed integer programming model for the operation of an experimental low-voltage network," in 2017 IEEE Manchester PowerTech, 2017.

Stefano Lilla (S'16) received the M.Sc. degree in electrical engineering in 1996 and the $\mathrm{Ph}$.D. degree in biomedical, electrical and systems engineering in 2019 from the University of Bologna, Italy. His research interests include microgrid 
energy management systems (control and optimization), distributed generation, renewable energy sources and battery energy systems. Dr. Lilla is consultant and project engineer in the renewable source area. He has designed and managed the construction of many dozens of photovoltaic, mini-hydro and concentrating solar systems.

Camilo Orozco (S'18) received the B.Sc. degree in Electronic Engineering from the Pontifical Xavierian University, Bogotá, Colombia, in 2008, and the M.Sc. degree in Sustainable Electrical Energy Supply from the University of Stuttgart, Stuttgart, Germany in 2017. Currently, he is pursuing his Ph.D. in electrical engineering at the University of Bologna, Bologna, Italy, in the framework of the European project INCITE. His research interests include decentralized and distributed control approach of micro-grid's components, local energy communities, integration of vehicle-to-grid (V2G) services and optimization modelling.

Alberto Borghetti (M'97-SM'03-F'15) received the Laurea degree in electrical engineering (Hons.) from the University of Bologna, Bologna, Italy, in 1992. Since then he has been working with the power system group of the same University, where is now a Professor of Electrical Power Systems. His research and teaching activities are in the areas of power system analysis, power system restoration after blackout, electromagnetic transients, optimal generation scheduling, and distribution system operation. He has received the Int. Conf. on Lightning Protection (ICLP) Scientific Committee Award in 2016 and the 2018 CIGRE Technical Council Award for SC C4 (System Technical Performance). From 2010 to 2017 he served as an Editor of IEEE Trans. on Smart Grid. Currently he is serving as an Editor of IEEE Trans. on Power Systems, a member of the Editorial Advisory Panel of EPSR, an Associate Editor of MPCE, and as Editor-in-Chief of Electrical Engineering - Archiv fur Elektrotechnik.

Fabio Napolitano (M'16-SM'17) is assistant professor at the Department of Electrical, Electronic and Information Engineering of the University of Bologna, Italy. From the same University, he received the M.S. degree (with hons.) in electrical engineering in 2003 and the Ph.D. degree in electrical engineering in 2009. His research interests are the analysis of power systems transients, in particular those due to indirect lightning strokes, and lightning protection systems. He is member of the Technical Committee 81 of CEI and Associate Editor of Electrical Engineering.

Fabio Tossani (S'15-M'16) received the B.S. (Hons.), M.S. (Hons.) and Ph.D. degree in electrical engineering from the University of Bologna, Italy, in 2010, 2012 and 2016, respectively. He is currently a junior assistant professor of the of the Power Systems Laboratory of the Department of Electrical, Electronic and Information Engineering "Guglielmo Marconi" of the University of Bologna, Italy. His research interests are power system transients, with particular reference to lightning electromagnetic pulse interaction with electrical networks, power systems protection and the integration of renewables in power distribution networks. He is assistant editor of the Electric Power Systems Research international journal. 